\title{
Molecular requirements for doxorubicin-mediated death in murine oocytes
}

\author{
A Jurisicova ${ }^{1,2,3}$, H-J Lee ${ }^{1}$, SG D’Estaing ${ }^{2,3}$, J Tilly ${ }^{1}$ and \\ GI Perez ${ }^{*}, 1$ \\ 1 Vincent Center for Reproductive Biology, Vincent Obstetrics and Gynecology \\ Service, Massachusetts General Hospital/Harvard Medical School, Boston, \\ MA 02114, USA \\ 2 Samuel Lunenfeld Research Institute, Mount Sinai Hospital, Toronto, Ontario \\ M5G 1X5, Canada \\ ${ }^{3}$ Departments of Physiology and Obstetrics and Gynecology, University of \\ Toronto, Toronto, Ontario M5G 1X5, Canada \\ * Corresponding author. Current address: GI Perez, Department of Physiology, \\ Michigan State University, 4173 BPS, East Lansing, Ml 48824, USA. \\ Tel: + 1-517-355-6475 ext 1158; Fax: + 1-517-355-5125; \\ E-mail: perezg@msu.edu
}

Received 10.5.05; revised 28.9.05; accepted 17.10.05; published online 27.1.06 Edited by R De Maria

\section{Abstract \\ We previously published evidence that oocytes exposed to doxorubicin (DXR), a widely used chemotherapeutic agent, rapidly undergo morphological and biochemical changes via discrete effector signaling pathways consistent with the occurrence of apoptosis. In this report, we elucidated the molecular requirements for actions of this drug in oocytes. Our results indicate that within $1 \mathrm{~h}$ of exposure DXR causes rapid DNA damage, and commits the oocyte to cytoplasmic fragmentation by the fourth hour, followed by delayed oocyte activation and execution of cytoplasmic fragmentation. Inhibitors that interfere with oocyte activation consistently rescue cytoplasmic fragmentation, but fail to suppress DNA damage. There was evidence of depletion of Bax, Caspase-2, $M A-3$ and $B c /-x$ transcripts, suggesting that modulations by DXR caused recruitment of these maternal transcripts into the translation process. Further- more, sphingolipids such as sphingosine-1-phosphate and ceramide modulate DXR actions by, respectively, altering its intracellular trafficking, or by sustaining the drug's contact with DNA. \\ Cell Death and Differentiation (2006) 13, 1466-1474. doi:10.1038/sj.cdd.4401819; published online 27 January 2006}

Keywords: oocyte; doxorubicin; apoptosis; gene expression; sphingolipids

Abbreviations: AA, $\alpha$-amanitin; ASMase, acid sphingomyelinase; $\mathrm{CB}$, cytochalasin $\mathrm{B}$; $\mathrm{CHX}$, cycloheximide; DXR, doxorubicin; hCG, human chorionic gonadotropin; KO, knockout; MAPK, mitogen-activated protein kinase; MEK, mitogen-activated protein kinase kinase; S1P, sphingosine-1-phosphate; NOC, nocodazole; RFU, relative fluorescence units; P-gp, P-glycoprotein; VEH, vehicle control; WT, wildtype

\section{Introduction}

The ovulated unfertilized oocyte in several species is known to decay with time. It is now clear that this spontaneous decay process in vivo as well as in vitro involves cellular fragmentation and ultimately death. ${ }^{1-3}$ Cytoplasmic fragmentation has also been observed in oocytes and embryos after several in vitro paradigms. ${ }^{4,5}$ Despite the fact that spontaneous fragmentation of the unfertilized oocyte has been documented almost three decades ago, very little is known about the cellular and molecular mechanisms governing this process.

Several reports proposed that the characteristics of cellular fragmentation in both oocytes and embryos are consistent with activation of programmed cell death. This was based on the presence of many hallmarks of the apoptotic pathways including DNA cleavage, activation of caspases, translocation of phosphatidyl serine and cytoplasmic condensation. ${ }^{6}$ It therefore seems paradoxical that cellular fragmentation is not described in several publications detailing induced activation of apoptosis in oocytes and/or zygotes. ${ }^{7-9}$ It is unclear in those papers whether the induction agent(s) failed to trigger cytoplasmic fragmentation or whether the researches refrained from reporting this observation. In the process of our work to elucidate the mechanisms of chemotherapy-induced oocyte depletion, we observed that doxorubicin (DXR) (anthracycline-derived chemotherapeutical agent) induces both cellular and DNA fragmentation of murine oocytes ${ }^{3,10}$ and thus represents an ideal model to study molecular events associated with cell death in oocytes.

In somatic cells, DXR is a potent activator of apoptosis, owing its actions to its ability to modulate several basic cell functions consequential to its: binding to DNA (by intercalation or crosslinking); generation of free radicals, leading to DNA damage and lipid peroxidation; inhibition of enzymes (helicases and toposoimerase II) important for DNA replication and transcription. Direct membrane effects have also been associated with DXR treatment. ${ }^{11}$ All these actions profoundly affect DNA function in exposed cells; by provoking erroneous transcription and/or replication, and eventually causing demise of the cell.

Although in the majority of cell types direct mediation of DNA damage is believed to be the specific target of DXR, ${ }^{12,13}$ it is worth mentioning that, in some other cell types, there is the presence of specific plasma membrane receptors for this drug and evidence of membrane-mediated effects. ${ }^{14}$ Moreover, there is also evidence for cytoplasmic action of DXR. ${ }^{15,16}$ Some of the drug derivatives have been shown to directly facilitate cytochrome $c$ release from mitochondria, thus unleashing apoptosome-dependent cell death, and bypassing DNA damage-induced apoptotic cascade. ${ }^{17}$ Other DXRinduced cytoplasmic events include hydrolysis of sphingomyelin and formation of ceramide, leading to activation of death; and depending on cell type, this activation may not always be $p 53$ dependent. Ceramide actions can also lead to 
JNK activation, ${ }^{18}$ Akt degradation and downregulation of the protein kinase B-dependent survival pathway. ${ }^{19}$

Unlike the situation of somatic cells, DXR mechanism(s) of action remains for the most part unknown in female germ cells (oocytes). In our first report on DXR-mediated death of oocytes, we analyzed data on the importance of several cell death regulatory pathways using mouse knockout (KO) or inhibitor models. Those experiments confirmed the importance of Bax, Caspase-2 and ceramide, but excluded p53 and Caspase-3 as important effectors in cytoplasmic fragmentation. ${ }^{3,10,20-22}$ In the current report, we attempted to further dissect some mechanistic aspects of the molecular pathways underlying cytoplasmic fragmentation of oocytes during the course of treatment with DXR, including: requirements of spindle checkpoints; exit from meiosis; analysis of gene expression; as well as the role of sphingolipids.

The data show that DNA damage and cytoplasmic fragmentation in oocytes are two independent events. Although DXR-induced cytoplasmic fragmentation is a late event, requiring de novo protein synthesis and exit from meiosis followed by oocyte activation; DNA damage on the other hand, occurs early and could not be suppressed by a variety of inhibitors of cellular fragmentation. Both cytoplasmic fragmentation and DNA damage can be prevented by interfering with DXR trafficking and/or the duration of its association with DNA; these actions appear to be mediated by the sphingolipids sphingosine-1-phosphate (S1P) and ceramide.

\section{Results}

\section{DXR induces early DNA damage, late activation and fragmentation}

In this study, as in our previous report, ${ }^{10} \mathrm{DXR}$ induced high rate of oocyte fragmentation $(53 \pm 4.8 \%, n=397 ; P<0.001)$, whereas spontaneous rate of cytoplasmic fragmentation was low in unexposed oocytes $(6.4 \pm 2.5 \%, n=414)$.

Regular assessment of cellular morphology every $2 \mathrm{~h}$ during a 24-h period established that oocyte fragmentation was preceded by activation (exit from meiosis) of the oocyte, as determined by extrusion of the second polar body and appearance of a single pronucleus (Figure 1b). Within 30 min from appearance of the pronucleus, ruffling of the oolema (plasma membrane) with partial fragmentation into large cellular structures was evident, followed by complete cytoplasmic fragmentation within an hour (Figure 1c).

When oocyte classification was based on time to formation of pronuclei post-DXR exposure (16-18th hour), only the ones that activated (e.g., exhibited formation of pronucleus) underwent subsequent cellular fragmentation. The minimal time that DXR required for induction of discernable cytoplasmic fragmentation was determined to be $4 \mathrm{~h}$ of continuous exposure, and the incidence of fragmentation did not increase with prolonged exposure up to $24 \mathrm{~h}$. The minimal period was as adequate as a $24-\mathrm{h}$ time of exposure $(54 \pm 6.72 \%, n=62$; compared to $53 \pm 4.8 \%, n=397)$ in inducing cellular fragmentation. Lesser exposure times (1, 2 or $3 \mathrm{~h}$ ) only elicited low levels of fragmentation (data not shown).
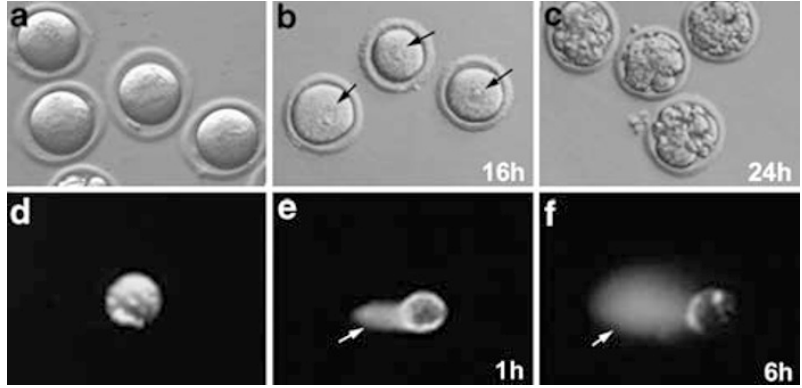

Figure 1 DXR causes delayed activation of oocytes and initiates rapid DNA damage. (a) Control; unexposed metaphase II oocytes. (b) Activated oocytes exhibited single pronuclei around $16 \mathrm{~h}$ after DXR exposure. (c) Fragmented oocytes; extensive cytoplasmic fragmentation was apparent $24 \mathrm{~h}$ after DXR exposure. (d) Intact DNA of unexposed oocyte. (e) DNA damage, evidenced by presence of comets, could be easily detected by $1 \mathrm{~h}$ after DXR exposure. (f) DNA damage was still evident by the sixth hour of exposure; and it remained relatively unchanged until the end of the 24-h culture period. Black arrows, pronuclei; white arrows, comets denoting DNA damage

Interestingly, the time for induction of oocyte cytoplasmic fragmentation was longer when contrasted to the rapid onset of DNA damage, which was easily detected within the first hour of exposure $(59 \pm 2.54 \%$ intact DNA, $41 \pm 0.47 \%$ cleaved DNA, $n=43$; Figure 1e) and remained detectable through the time course of exposure (Figure 1e and f). Removal of oocytes from exposure to DXR at any time point after $6 \mathrm{~h}$ had no effect on the extent of DNA damage, which remained relatively unchanged beyond this hour $(92 \pm 5.22 \%$ cleaved DNA, $6.4 \pm 2.45 \%$ intact DNA, $n=35$ ) up to the end of the 24-h culture period $(95 \pm 3.48 \%$ cleaved DNA, $3.41 \pm 0.93 \%$ intact DNA, $n=52$ ). Thus, DNA damage triggered by DXR is rapid and cannot be spontaneously repaired even after premature withdrawal of oocytes from the treatment.

\section{Cytoplasmic fragmentation requires protein synthesis and exit from meiosis}

In order to establish the cellular events required for complete execution of cell death in oocytes, we used different inhibitors known to interfere with individual components of cell cycle progression and molecule turnover. Treatment of oocytes with the RNA polymerase II inhibitor, $\alpha$-amanitin (AA), had no effect on the rate of spontaneous cytoplasmic fragmentation (vehicle control (VEH) $4.5 \pm 1.6 \%, n=240$; AA $4.67 \pm 4.67 \%$, $n=34$ ), or that induced by DXR (DXR $64 \pm 2.92 \%, n=221$; AA/DXR $58.3 \pm 6.01 \%, n=75)$. However, the protein synthesis inhibitor, cycloheximide ( $\mathrm{CHX})$, abrogated both spontaneous and DXR-induced fragmentation ( $\mathrm{CHX} \mathrm{0 \% ,} n=34$; $\mathrm{CHX/DXR} \mathrm{5.8 \pm 2.71 \% ,n=120;}$ DXR $64 \pm 2.92 \%, n=221$; $P<0.001$ ), indicating a need for de novo protein synthesis. Results of these inhibitory experiments are summarized in Figure 2a.

To separate the nuclear and cytoplasmic events responsible for oocyte fragmentation, we first determined whether the oocyte requires exiting from meiosis and entering a mitotic cell cycle in order to execute cellular fragmentation. Consistent with our hypothesis, destabilization of the spindle with nocodazole (NOC) did not permit the treated oocytes to exit 

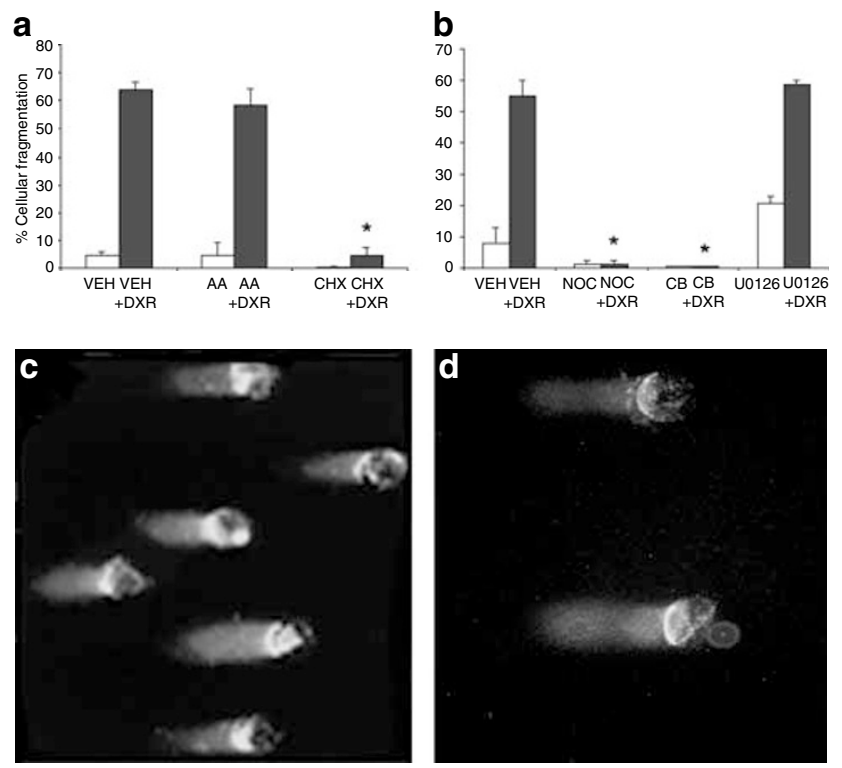

Figure 2 DNA damage and cytoplasmic fragmentation in oocytes are two independent events. (a, b) Rates of cytoplasmic fragmentation (as \% of all exposed cells) in control (open bars) and DXR-exposed (filled bars) oocytes. Although AA and U0126 had no effect on DXR-induced fragmentation, CHX, or NOC, or CB almost completely abolished cytoplasmic fragmentation. (c) DNA damage evidenced by comet tails was extensive in fragmented oocytes $24 \mathrm{~h}$ from initiation of culture; and (d) it could not be prevented by NOC (shown here), or $\mathrm{CHX}$, or AA. ${ }^{*} P<0.05 ;{ }^{* *} P<0.001$

meiosis, resulting in almost total inhibition of DXR-mediated cytoplasmic fragmentation (DXR 44.6 $\pm 5.24 \%, \quad n=176$; NOC/DXR 1.25 $\pm 1.25, n=91 ; P<0.001$; Figure $2 b)$. NOC treatment blocked the oocyte in metaphase II with chromosomal plate localized on one pole (Figure $3 c$ and $d$ ). Therefore, depolymerization of the spindle activated a checkpoint, which prohibited the oocyte from progressing through the mitosis-like cycle, and thus prevented activation and cellular fragmentation (Figures $2 b$ and $3 d$ ).

To further elucidate whether additional checkpoints exist upon exiting from meiosis, oocytes were treated with an inhibitor of actin polymerization, cytochalasin B (CB). As expected, CB allowed oocytes to exit from meiosis and did not interfere with formation of pronuclei (Figure $3 e$ and $f$ ), but it completely inhibited spontaneous and DXR-mediated cytoplasmic fragmentation (DXR 44.6 $\pm 5.24 \%, n=176$; CB/DXR $0 \%, n=56 ; P<0.001$; Figure $2 \mathrm{~b}$ ). As mitogen-activated protein kinase (MAPK) activity is responsible for maintenance of meiotic arrest, we explored its role in the DXR-mediated death. The mitogen-activated protein kinase kinase (MEK) inhibitor, U0126, which interferes with MAPK activity did not significantly alter response of oocytes to DXR (DXR 44.6 $\pm 5.24 \%, n=176 ; \quad U 0126 / D X R \quad 58.7 \pm 1.57 \%, n=66$; $P>0.05$; Figure 2b), whereas it increased $(P<0.001)$ spontaneous rate of fragmentation (VEH $8.18 \pm 3.36 \%$, $n=174$; U0126 20.68 $\pm 2.22 \%, n=66$; Figure $2 b$ ).

Suppression of DXR-induced death by some of the inhibitors used in these experiments could have been achieved via rearrangements of cytoskeletal components or as a consequence of preventing DXR-induced DNA damage. Therefore, we next performed comet assays on oocytes
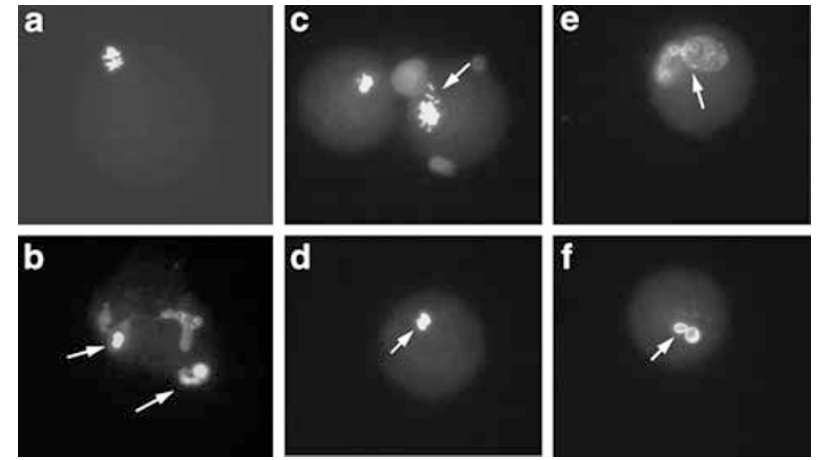

Figure 3 Staining of oocyte chromatin by DAPI after $24 \mathrm{~h}$ in culture. (a) Unexposed oocyte, maintaining metaphase II arrest. (b) Oocytes exposed to DXR exhibited signs of cellular fragmentation, partial DNA condensation (arrows) and total chromatin disintegration. (c) Oocytes exposed to NOC (alone) could not exit from meiosis. (d) Oocytes exposed to NOC/DXR maintained chromosomal plate (arrow) and cellular integrity. (e) CB (alone) allowed oocyte exit from meiosis, but prevented exclusion of second polar body, leading to formation of two pronuclei (arrow). (f) CB allowed oocyte activation after DXR exposure; as evidenced by presence of two pronuclei with heavily condensed chromatin (arrow), but prevented cytoplasmic fragmentation

exposed to each one of the studied inhibitors (AA, CHX, NOC or $\mathrm{CB}$ ) without or with $\mathrm{DXR}$ to analyze the integrity of genomic DNA. Despite the observations that $\mathrm{CHX}, \mathrm{CB}$ and NOC very efficiently obliterated DXR-induced cytoplasmic fragmentation, none of the inhibitors prevented the accompanying DNA damage $(95 \pm 4.8 \%$ cleaved DNA; at least 40 oocytes/group; three different experiments). Figure $2 \mathrm{c}$ and $\mathrm{d}$ shows representative comets after $24 \mathrm{~h}$ of incubation, respectively, with DXR or DXR + NOC. Chromatin morphology in oocytes exposed to CB/DXR displayed condensed and shrunken morphology (Figure 3f), but did not undergo any visible chromatin disintegration (nuclear fragmentation) as frequently observed when DXR is added alone (Figure $3 \mathrm{~b}$ ). However, the DNA damage as determined by the comet assay was extensive $(96 \pm 10.12 \%$ cleaved DNA).

\section{DXR causes depletion of transcripts for various cell death genes}

The ovulated oocyte is believed to be in a transcriptionally silent stage, as chromosomes are condensed and thus inaccessible to the transcriptional machinery. Assessment of transcript level in these oocytes may therefore gives an insight into $\mathrm{mRNA}$ recruitment and thus may reveal the degree of their utilization. In order to determine which genes may be important players in the regulation of cellular fragmentation during the time course of DXR treatment, we proceeded to assess the accumulation of polyadenylated mRNAs in oocytes. The most dramatic differences were observed by the sixth hour of DXR exposure. In all the cases as shown in Figure $4 \mathrm{a}$, we detected reduction in polyA transcript levels for Bax ( 30\%), Caspase-2 ( 30\%), MA-3 ( 45\%) and Bag-1 $(45 \%)$. Minor to undetectable changes were documented in the levels of transcripts for $\mathrm{Bcl}-2, \mathrm{Bcl}-\mathrm{w}, \mathrm{Dad}-1$ and $p 53$ throughout the treatment period (data not shown). Interestingly, transcript of $\mathrm{Bcl}-x$ was also downregulated ( $\sim 5 \%$ ), but this was only apparent by the 12th hour of exposure 
a
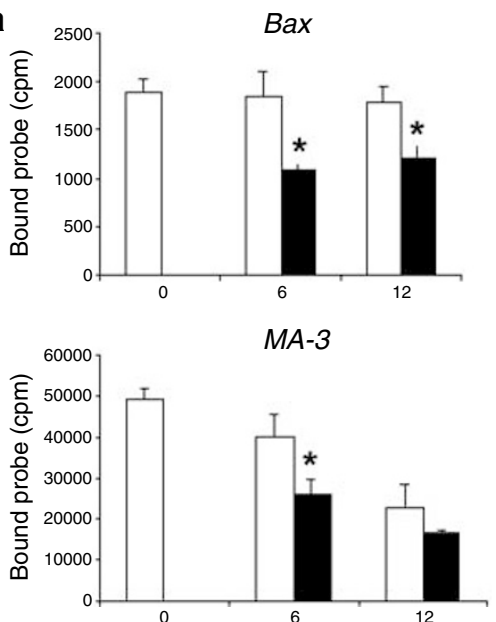

b

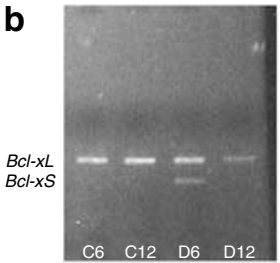

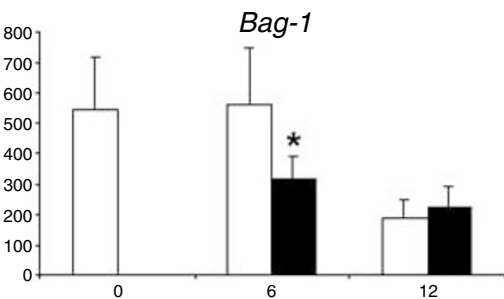

$B c l-x$
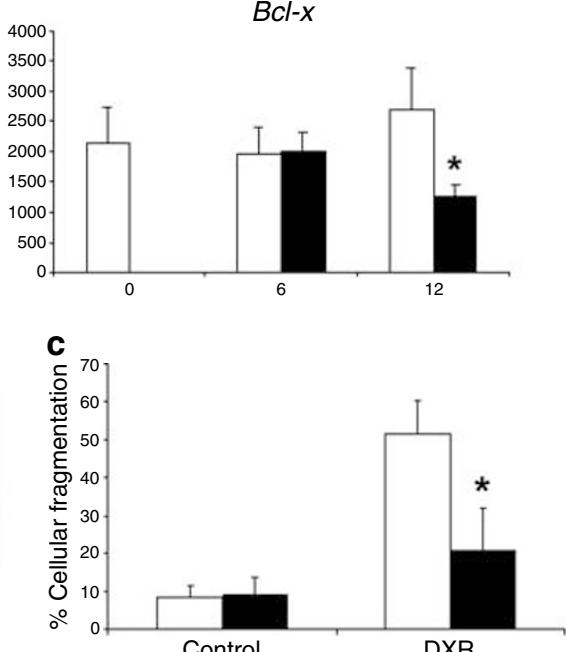

Figure 4 DXR causes depletion of transcripts for various cells death genes, and microinjection of BCl-X $\mathrm{X}_{\mathrm{LC}}$ prevents DXR-induced oocyte apoptosis. Pattern of gene expression in control (opened bars) or in oocytes exposed to DXR (filled bars) at various time points $(0,6,12$; in hours) after initiation of culture. Level of transcript accumulated is expressed in counts per minute (cpm) of bound probe generated for 5 pools of oocytes: (a) Bax, Bag-1, MA-3 and Bcl-x. (b) Changes in Bcl-x isoform profile caused by DXR, RT-PCR (left) and Southern blot (right) for BCl-x isoforms after indicated time points. (c) Microinjection of recombinant Bcl- $\mathrm{X}_{\mathrm{L} \Delta \mathrm{C}}$ (filled bar) into oocytes decreases the percentage of DXR-induced oocyte apoptosis compared with the buffer-injected group (open bar) $24 \mathrm{~h}$ after DXR exposure. ${ }^{\star} P<0.05$

(Figure 4a). Concomitantly, appearance of $B c /-x_{S}$ transcript was noted in samples exposed to DXR (Figure $4 b$ ), although this was observed at different time points in different batches of oocytes. In order to determine whether alteration of $B c /-x_{L}$ to $B c l-x_{S}$ ratio can contribute to DXR-mediated death, we microinjected $\mathrm{Bcl}-\mathrm{x}_{\mathrm{L} \Delta \mathrm{C}}$ (antiapoptotic $\mathrm{Bcl}-\mathrm{x}_{\mathrm{L}}$ isoform) recombinant protein, or vehicle (buffer) into the oocytes before DXR treatment. Although baseline levels of apoptosis were not affected by elevated $\mathrm{Bcl}-\mathrm{x}_{\mathrm{L}}$ levels, DXR-mediated death was significantly decreased by $\sim 50 \%(P=0.0044)$ following microinjection of this protein (Figure $4 c$ ).

\section{S1P alters intracellular trafficking of DXR in vitro}

We have previously documented that S1P (a ceramide metabolite) prevents cellular as well as DNA fragmentation in oocytes exposed to DXR in vitro. ${ }^{10,20}$ Because sphingolipids play key roles in the regulation of several fundamental biological events including uptake and movement of molecules within the cells, ${ }^{23}$ we next assessed the role of S1P in modulating the intracellular trafficking of DXR. By exploiting the autofluorescent nature of DXR, we monitored its accumulation in both the cytoplasm and on the chromatin by computer-aided analysis of fluorescence intensity using deconvolution microscopy. When oocytes were cultured in the presence of S1P this caused a retardation in the accumulation of DXR in the cytoplasm (Figure $5 \mathrm{a}-\mathrm{c}$ ) as well
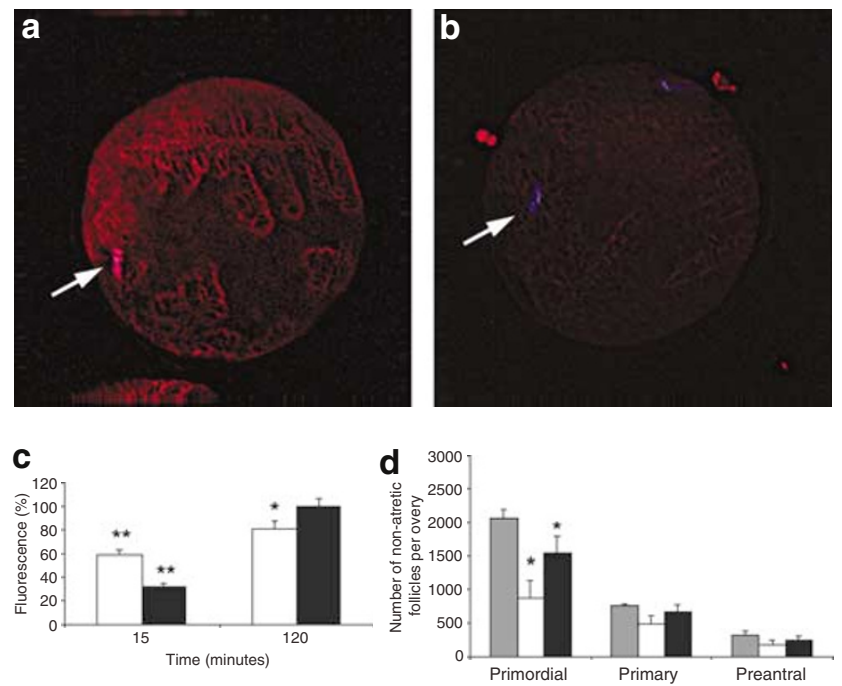

Figure 5 S1P mediates delay of DXR accumulation in ovulated oocytes and prevents primordial follicle death in vivo. DXR accumulation as reflected by the autofluorescent nature of this compound in cytoplasm (red) and on DNA (red + blue = purple): (a) DXR alone; (b) S1P/DXR; pretreatment with S1P delayed DXR accumulation in both cytoplasm and DNA. (c) Relative decrease in fluorescence in cytoplasm (open bars; $n=20$ ) or on DNA (filled bars; $n=10$ ) 15 and 120 min after initiation of treatment (S1P/DXR); compared with DXR alone $(100 \%)$. (d) Assessment of ovarian reserve $(n=3)$ after in vivo exposure to DXR. S1P prevented depletion of primordial follicles, assessed $24 \mathrm{~h}$ after administration of Vehicle (hatched bars), or DXR (opened bars), or S1P/DXR (filled bars). ${ }^{\star \star} P<0.001 ;{ }^{*} P<0.05$ 
as in the DNA (Figure 5a-c). By $15 \mathrm{~min}$ of exposure, the total DXR content in the cytoplasm/per oocyte in the S1P/DXR group $(n=19)$ was depressed by $42 \%(P<0.001$; Figure $5 c)$ relative to DXR treatment alone $(n=20)$. Furthermore, there was also depressed accumulation of DXR on the DNA by $68 \%$ (S1P/DXR, $n=10 ; P<0.001$; Figure $5 \mathrm{c}$ ). After $120 \mathrm{~min}$ of exposure, S1P still continued to significantly retard the accumulation of total DXR in the cytoplasm by $20 \%(n=19$; $P<0.05$; Figure 5c), but was no longer effective at preventing its binding to DNA.

To rule out the possibility that S1P protection of oocytes from DXR-induced death was not just an in vitro response, we explored the efficiency of this compound under in vivo conditions. Quantitative assessment of the ovarian follicle pool, based on follicle counts, showed that a single intrabursal injection of S1P, given $3 \mathrm{~h}$ before DXR treatment, partially protected the primordial follicle pool from chemotherapyinduced death (Figure 5d).

\section{Ceramide facilitates DXR actions by sustaining DXR/DNA contact}

To assess the functional role of ceramide in DXR trafficking and/or retention, we examined oocytes of ASMase-null mice, which are chronically deficient in ceramide generation. ${ }^{24}$ Previously, we have also shown that oocytes from these mice are resistant to DXR-induced cellular as well as DNA fragmentation. ${ }^{20}$

Surprisingly, oocytes of ASMase-null mice internalized DXR faster $(295.03 \pm 3.64$ relative fluorescence units (RFU) in the cytoplasm, $n=30 ; P<0.001$; Figure $6 \mathrm{~b}$ ) than wild-type (WT) oocytes $(110.7 \pm 3.29 \mathrm{RFU}$ in the cytoplasm, $n=30$; Figure 6a). By $15 \mathrm{~min}$ of exposure, DXR was mostly localized to the chromatin in the KO oocytes $(476.93 \pm 6.56 \mathrm{RFU}$ in DNA, $n=30 ; P<0.001$ ), whereas only one-third of the DXR fluorescence could be detected in the chromatin of WT $(128 \pm 3.27$ RFU) oocytes $(n=30)$. After $120 \mathrm{~min}$ upon DXR removal, there was no difference in the amount of drug remaining in the cytoplasm of KO and WT oocytes. However, DXR was barely detectable on the chromatin of KO oocytes $(63.63 \pm 1.6 \mathrm{RFU}$ in DNA; $n=30 ; P<0.001$; Figure $6 \mathrm{~d})$ in comparison to $442.67 \pm 8.37 \mathrm{RFU}$ in the WT oocytes $(n=30$; Figure $6 c$ ). Thus, oocytes from WT mice retained DXR on the chromatin for up to $24 \mathrm{~h}$ despite the brief exposure to this drug.

\section{Discussion}

Our results not only clearly indicate that, DNA damage and cytoplasmic fragmentation are two distinct events leading to cell death but also that, both have differential molecular requirements. As both of these hallmarks of cell death occurred sequentially during exposure to the same factor (DXR), it could be assumed that they are regulated by shared molecular pathways. However, based on our data this does not appear to be the case, because DNA damage was almost instantaneous, while the decision to undergo cytoplasmic fragmentation required at least $4 \mathrm{~h}$ of DXR exposure. Considering that the incidence of fragmentation did not increase beyond $4 \mathrm{~h}$ of exposure to DXR, we reached the
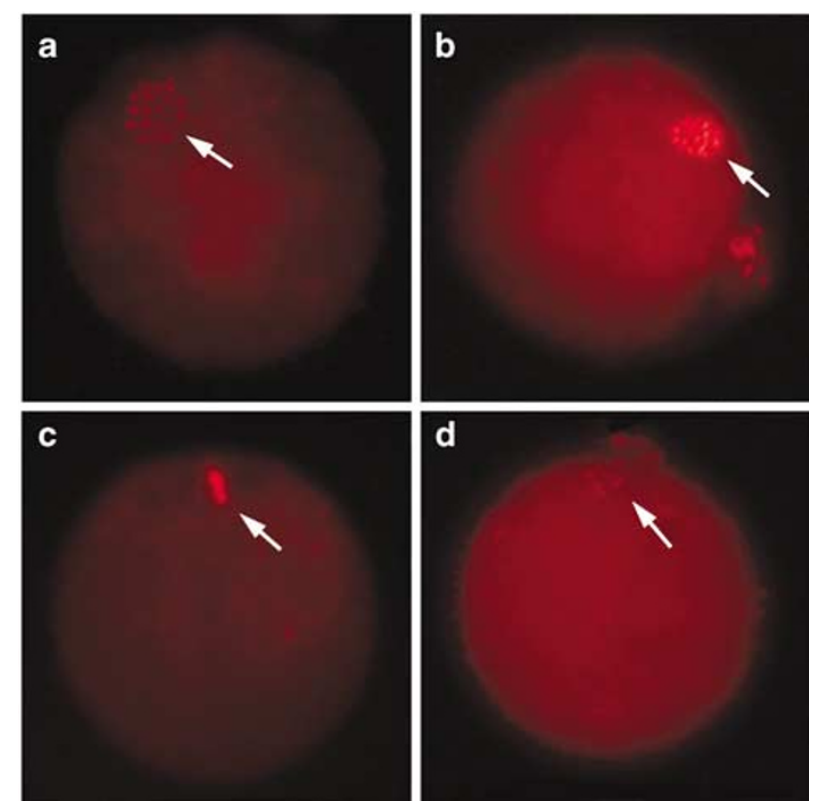

Figure 6 ASMase-null oocytes exhibit altered DXR/DNA retention. In comparison to WT oocytes (a), accumulation of DXR in the cytoplasm was faster in oocytes lacking ASMase (b); $15 \mathrm{~min}$ after initiation of culture. Surprisingly, while WT oocytes retained DXR bound to DNA $2 \mathrm{~h}$ after withdrawal (c), DXR failed to remain attached to chromatin in ASMase-null oocytes (d)

conclusion that commitment to cellular fragmentation occurred around the fourth hour of oocyte exposure to the drug.

Before the initiation of ocyte fragmentation, its activation was necessary, an observation that was made possible by regularly assessing the status of the oocytes every $2 \mathrm{~h}$. However, oocyte activation was not completely synchronous, as individual oocytes continued to enter activation status from the 16th hour until the 23rd hour postexposure to DXR. This inclination probably reflects the maturity stages of the follicles at the time of cycle synchronization.

Interestingly, it is also clear that female gametes do possess late checkpoints for DNA damage. These checkpoints are only activated after exiting from meiosis, as oocytes with highly fragmented DNA are capable of completing meiosis, extruding polar body, decondensing into normally appearing pronucleus, and only then commit suicide. These observations are in agreement with previous reports, which described similar behavior by oocytes that sustained DNA damage. $^{25}$

We also point out that chromatin behavior is also likely regulated by two distinct pathways, one orchestrates DNA damage and the other facilitates nuclear fragmentation. This theory is based on the observation that whereas chromosomes exhibited DNA damage, which could not be prevented by NOC and $\mathrm{CB}$, chromatin disintegration (nuclear fragmentation) was completely inhibited by these compounds. These observations are also consistent with the existence of two independent events regulating nuclear breakdown in rat blastocyst exposed to high levels of glucose. ${ }^{26}$

With respect to molecular pathways responsible for governing cell death decisions, it is clear that the oocyte is unique, as it is the only cell in the mammalian body arrested at 
the metaphase II of meiosis. The inhibitory studies revealed that in our in vitro oocyte model, de novo transcription is not required for activation of cytoplasmic fragmentation; however, de novo protein production was mandatory as treatment with $\mathrm{CHX}$ completely abrogated induction of cytoplasmic fragmentation. As it has been previously determined that $\mathrm{CHX}$ becomes ineffective during later time points of treatment, ${ }^{27}$ it is likely that proteins required for the activation of fragmentation are synthesized within the early stages of exposure to DXR. This is consistent with our observation that $4 \mathrm{~h}$ of minimum exposure to DXR is required before commitment to death pathways. Moreover, these observations also suggest that cytoplasmic fragmentation in oocytes is regulated by maternal products accumulated during oogenesis.

A lack of transcription coupled with the necessity for de novo protein synthesis led us to speculate that availability of transcripts for genes that are required for cell death in the oocyte are altered during the time course of DXR treatment. The results suggest that the decrease in the polyA transcript levels may reflect utilization of these transcripts in protein turnover. This picture would be consistent with the increased accumulation of cell death inducing proteins associated with commitment to death in somatic cells, ${ }^{19}$ and in germ cells with advanced age. ${ }^{24}$ Recently, we have also showed that injection of recombinant Bax protein into healthy oocytes triggers fragmentation, ${ }^{20}$ confirming the importance of this protein in controlling the survival rheostat in ovulated oocytes. Thus, depletion of polyA mRNA for Bax and Caspase-2 by the sixth hour after DXR exposure, preceded by commitment to fragmentation by the fourth hour of treatment, corroborated the observed natural set of events leading to death in treated oocytes.

We also evaluated gene transcripts whose importance in the oocyte fragmentation is presently unknown. Of these, we find it interesting that total $\mathrm{BCl}-x$ levels appeared reduced by the 12th hour time point, accompanied by a switch in isoform splicing, resulting in expression of $B c /-x_{S}$. We have previously shown upregulation of $B \mathrm{Bl}-x_{S}$ transcript in some murine oocytes/zygotes undergoing spontaneous fragmentation in vivo; and in human fragmented embryos in vitro. ${ }^{28,29}$ Variable increase in the $\mathrm{Bcl}-x_{S}$ transcript in the DXR-treated groups could be explained by the asynchrony of the oocytes in the induction of cell death pathway; this was reflected by lack of synchrony in cytoplasmic fragmentation, and as explained earlier, it was initiated by some oocytes as early as the 17th hour, and as late as $24 \mathrm{~h}$ post-DXR exposure. Such degrees of deviations are expected, as we used an outbred mouse strain, and consequently there is bound to be a certain degree of variability in the 'genetic make-up' among individual oocytes. We were therefore not surprised to detect variable proportions of $\mathrm{Bcl}-x_{L}$ and $\mathrm{Bcl}-x_{S}$ in the different pools of oocytes. As the oocyte accumulates maternal untranslated RNAs, the mechanism of $B c /-x_{S}$ transcript increase is most likely due to regulation of $\mathrm{Bcl}-x$ pre-mRNA splicing and not due to de novo transcription, since transcriptional inhibitors were not capable of suppressing oocyte death. Switching the ratio in favor of $B c l-x_{L}$ appears to be critical for the oocyte to survive the DXR insult. Further studies addressing the importance of $B c /-x$ splicing in the activation of oocyte and embryo fragmentation are currently ongoing in our laboratory.
It is also important to note that, not all transcripts were altered after DXR exposure. Lack of $p 53$ transcript recruitment observed in the present study as well as its dispensability in the DXR-mediated death reported previously, ${ }^{10}$ further supports the hypothesis that, other members of p53 surveillance proteins may be involved in DXR modulations. Discovery of two other p53 family members (p63 and p73) which share many molecular features of p53 apoptosis induction makes these molecules excellent candidates for modulating DXRmediated death pathways in oocytes.

During the course of the studies to decipher DXR effects on germ cells, we explored innumerable chemicals as antagonists of the drug effects, but so far, S1P is the only compound able to block both cellular and DNA fragmentation. ${ }^{20}$ In the current study, we show that cellular uptake and DNA retention of the chemotherapeutic drug are both impaired by interfering with intracellular levels of ceramide following treatment with its biological inhibitor S1P, or by knocking out ASMase. Thus, this scenario emphasizes the significance of phospholipids, particularly ceramide, as important regulators of these two cellular processes. Even so, further experiments are needed to fully determine the trafficking patterns of DXR without or with S1P. This would rule out the possibility that quenching of DXR fluorescence, a concern in the present studies, was not due to the interaction of the chemotherapeutic drug with lipids or other molecules in the intracellular environment. ${ }^{30}$

The observations we made using fluorescence microscopy in the present experiments also indicate that, the cytoplasm and chromosomal DNA of both DXR-sensitive (WT) and -resistant (ASMase-null) oocytes are primary targets for DXR. Moreover, early interference with either DXR uptake or DNA retention is crucial for the prevention of DNA damage induced by DXR. Nevertheless, the time that DXR is retained on the DNA appears to be more critical than its cytoplasmic localization in eliciting the relative drug effects. This is illustrated in the DXR-resistant ASMase-null oocytes, which after $2 \mathrm{~h}$ beyond removal from DXR possessed almost undetectable levels of the drug on the DNA, whereas they maintained considerable amounts of the chemotherapeutic drug in the cytoplasm. This faster efflux of DXR from the DNA in the ASMase-null oocytes when compared to WT oocytes, likely protects their DNA from damage, as these oocytes do not exhibit comets, even after $24 \mathrm{~h}$ of continuous exposure to the chemotherapeutic drug. ${ }^{20}$ These results are in agreement with studies in other cells where it has been shown that sensitivity to DXR is directly related to the nuclear localization of the drug ${ }^{12,13}$ and slower release from this site. ${ }^{31}$

Although a longer residence time of DXR on DNA might be desirable to increase toxicity to tumor cells, a display of overly high affinity and slow dissociation may also cause irreversible DNA damage in normal cells and loss of selectivity for tumor cells. Formation of relatively long-lasting DXR/DNA associations in WT oocytes might explain why in vivo, female germ cells are unselectively killed by the secondary effects of some chemotherapeutic chemicals.

In some cells, resistance to chemotherapy is usually accompanied by higher expression of P-glycoprotein (P-gp), which pumps cytoplasmic drug out of cells as it is released from the DNA. ${ }^{32}$ However, it is unlikely that this protein played a role in the current experiments, as there is no statistical 
difference between WT and null oocytes in the levels of DXR remaining in the cytoplasm $2 \mathrm{~h}$ after the drug's removal. It is not possible to compare our results to any previous work, as, to our knowledge, there are no published data on the levels of P-gp in ASMase-null oocytes.

Hence, based on our data we propose that ceramide might be directly interacting with DXR and helping it to maintain contact with the DNA, long enough until the activation of nuclease is sufficient. As cytoplasmic ${ }^{33}$ and nuclear localization of ceramide have been previously documented, ${ }^{34}$ we hypothesize that once inside the oocytes, DXR takes residence in the cytosol, binds to ceramide (if present), and ultimately it (DXR) or its derivative(s) is transported to the nucleus where again complexing with ceramide enhances its association with the DNA. As there is published evidence ${ }^{35}$ of the possible modulatory role of other lipids (e.g., sphingomyelin) in DXR mediations, we cannot exclude their contribution to the DXR pathway in this model. Experiments in our laboratory are currently in progress to address the molecular mechanism(s) involved and the impact on the intracellular trafficking/retention of DXR; investigations that ultimately may enhance our understanding of drug resistance and sensitivity.

Collectively the data show that DNA damage and cytoplasmic fragmentation in oocytes are two independent events. Although DXR-induced cytoplasmic fragmentation is a late event requiring de novo protein synthesis and exit from meiosis followed by oocyte activation, DNA damage on the other hand, occurs early and could not be suppressed by the tested inhibitors of cellular fragmentation. Both cytoplasmic fragmentation and DNA damage can be prevented by interfering with DXR trafficking and/or the duration of its association with DNA; these actions appear to be mediated, within a 2-h window, by the sphingolipids S1P and ceramide, respectively.

\section{Materials and Methods}

\section{Animals}

For all experiments except those involving in vivo treatment with DXR, or ASMase-null oocytes, female ICR mice were purchased from Taconic (Germantown, NY, USA) at 6-8 weeks of age. For the S1P treatment in vivo, C57BL/ 6 females 6 weeks of age were purchased from Charles River Laboratories (Wilmington, MA, USA). The generation and genotyping of ASMase mutant and WT mice has been detailed previously; ${ }^{20}$ we continue to breed the mice at our animal facilities by mating heterozygous animals. All experiments involving animals described herein were reviewed and approved by the institutional animal care and use committees of Massachusetts General Hospital and MSU.

\section{Oocyte collection and culture}

Female mice were superovulated with $10 \mathrm{IU}$ of equine chorionic gonadotropin (Professional Compounding Centers of America, Houston, TX, USA) followed by $10 \mathrm{IU}$ of human chorionic gonadotropin (hCG; Serono Laboratories, Norwell, MA, USA) $46 \mathrm{~h}$ later. Mature oocytes were collected from the oviducts $16 \mathrm{~h}$ after hCG injection. Cumulus cellenclosed oocytes were denuded of cumulus cells by a 1-min incubation in $80 \mathrm{IU} / \mathrm{ml}$ of hyaluronidase (Sigma, St. Louis, MO, USA), followed by three washes with culture medium.
All cultures were carried out in human tubal fluid (Irvine Scientific, Santa Ana, CA, USA) supplemented with $0.5 \%$ bovine serum albumin (fraction V; Gibco-BRL Life Technologies, Grand Island, NY, USA). Oocytes were cultured in $0.1 \mathrm{ml}$ drops of culture medium (10 oocytes/drop) under paraffin oil (Speciality Media, Phillipsburg, NJ, USA), and incubated for up to $24 \mathrm{~h}$ at $37^{\circ} \mathrm{C}$ in a humidified atmosphere of $5 \% \mathrm{CO}_{2}-95 \%$ air. The oocytes were assigned to treatment groups as planned, and incubations performed without or with freshly made DXR $(200 \mathrm{nM}$; Alexis Biochemicals, San Diego, CA, USA) or with various inhibitors as described later. Oocyte morphology was evaluated $24 \mathrm{~h}$ later. In a separate set of experiments, we determined the minimal time of exposure DXR required for eliciting cytoplasmic fragmentation. Oocytes were placed in medium containing DXR as described earlier; but, were removed after 1, 2, 3, 4, 5, 6 and $8 \mathrm{~h}$ of culture, washed thoroughly, and incubation continued in medium alone. Occurrence of cytoplasmic fragmentation was scored every $2 \mathrm{~h}$ for a total of $24 \mathrm{~h}$ from the start of drug exposure.

\section{Inhibitor studies}

In order to establish molecular requirements of DXR-mediated cytoplasmic fragmentation, we used several inhibitors of basic cellular functions. First, we determined whether oocytes contain all the necessary protein machinery to undergo cellular fragmentation upon exposure to DXR; for that we performed a series of experiments pertaining to: inhibition of transcription (AA, $100 \mu \mathrm{g} / \mathrm{ml}$ ); inhibition of translation (CHX, $20 \mu \mathrm{g} / \mathrm{ml}$ ); spindle destabilization (NOC, $10 \mu \mathrm{M}$ ); and inhibition of actin polymerization (CB, $5 \mathrm{mg} / \mathrm{ml}$ ). Lastly, we used the MEK inhibitor (U0126, $10 \mu \mathrm{M})$ to determine to which extent abrogation of MAPK signaling affects rates of cytoplasmic fragmentation upon exposure to DXR. In all cases, the appropriate inhibitor was added to the oocyte cultures 30 min before DXR. Each experiment was performed at least three times. All reagents were obtained from Sigma (St. Louis, MO, USA), unless otherwise stated.

\section{Characteristics of apoptosis}

At the end of the culture period, the oocytes were fixed, stained and evaluated, as detailed previously. ${ }^{3,10,20-22}$ We focused on describing characteristics of apoptosis: mainly morphological changes (e.g., condensation, budding and cellular fragmentation) and biochemical alterations (i.e., DNA cleavage using Comet Assay Kit, Trevigen, Gaithersburg, $M D, U S A)$. Analysis of the comets to generate quantitative data was performed using a computer-based program (Duty et al. ${ }^{36}$ VisComet; Impuls Computergestutzte Bildanalyse $\mathrm{GmbH}$, Gilching, Germany). The percentage of oocytes that underwent apoptosis out of the total number of oocytes cultured per drop in each experiment was then calculated.

\section{Transcript status}

Oocytes completely denuded of any cumulus cells were harvested right after collection (T0) or after culturing for 6,12 , and $24 \mathrm{~h}$ in medium alone (control) or in the presence of DXR. Gene expression was determined by a quantitative reverse transcription-polymerase chain reaction (RT-PCR)based assay as detailed previously. ${ }^{28}$ Briefly, CDNAs derived from pools of five oocytes were amplified as described by Brady and Iscove. ${ }^{37}$ Each time point was replicated 5 times. The amplified material was then analyzed by hybridization of dot blots with cDNA probes radiolabeled by randompriming, followed by quantitation of signals using a phosphorimager and a calibrated $\left[{ }^{14} \mathrm{C}\right]$ microscale (Amersham, Canada) for correcting times of exposure. Every sample was normalized against $\beta$-actin mRNA levels. 
Our survey included genes that have been previously implicated in the regulation of oocyte cytoplasmic fragmentation triggered by DXR, such as Bax and Caspase-2; genes dispensable for this pathway, p53, as well as genes whose functions were not directly assessed so far $(B c l-x, B c l-w$, Bag-1, MA-3).

The cDNA probes (bcl-w, bcl-2, bag-1, caspase-2, MA-3) were either donated from outside laboratories, or were cloned using probes recognizing the coding regions of $b c l-x, b a x, p 533^{28}$ For the latter, we used an oligo dT primed extra embryonic ectoderm cDNA library derived from $d 7.5$ murine embryos. ${ }^{28}$

As $B c l-x$ can be alternatively spliced into two proteins $\left(B c l-x_{L}\right.$ and $\mathrm{Bcl}-\mathrm{x}_{\mathrm{S}}$ ) with opposite functions, we also assessed the proportion and ratio of these variants at each time point. Briefly, we performed RT-PCR with specific primers spanning the alternative splice site of the $b c l-x$ gene. ${ }^{38}$ Aliquots of five oocytes were removed at harvesting, and after 6, 12 and $20 \mathrm{~h}$ of DXR exposure. Each aliquot was lysed in $50 \mu \mathrm{l}$ of GT solution, and total nucleic acid was recovered by ethanol precipitation using glycogen as a carrier, and RT-PCR was then performed as previously described. ${ }^{28}$ Each PCR cycle consisted of denaturation at $95^{\circ} \mathrm{C}$ for $60 \mathrm{~s}$, annealing at $56^{\circ} \mathrm{C}$ for $60 \mathrm{~s}$, and extension at $72^{\circ} \mathrm{C}$ for $90 \mathrm{~s}$. The expected sizes for the amplified products were $760 \mathrm{bp}$ for $B c l-x_{L}$ and $550 \mathrm{bp}$ for $B c l-x_{S}$. To confirm the identity of amplified products, Southern blot analysis was performed with a radiolabeled cDNA probe recognizing the shared coding region of both splice variants.

\section{Microinjection of oocytes}

Microinjection needles and holding pipettes were made using a Sutter puller (Sutter Instruments, Novato, CA, USA) and a De Fonbrune Microforge (EB Sciences, East Granby, CT, USA). The microinjection needles had inner diameters of $5 \mu \mathrm{m}$ with blunt tips. Recombinant $\mathrm{BCl}-\mathrm{x}_{\mathrm{L} \Delta \mathrm{C}}{ }^{39}$ kindly donated by Dr. Newmeyer, was dissolved in microinjection buffer to a concentration of $1.5 \mathrm{mg} / \mathrm{ml}$ and was aspirated into the needle by negative suction. The protein suspension or buffer ( $\sim 6 \mathrm{pl}$ per oocyte) was injected into oocytes using a Piezo micromanipulator. Oocytes that did not survive the microinjection procedure (routinely about $30 \%$ ) were discarded, and the remaining oocytes were randomly allocated into control or DXR treatment group. Assessment of apoptosis was performed $24 \mathrm{~h}$ later.

\section{S1P experiments in vivo}

Young adult ( 6 weeks of age) female mice were anesthetized, and a dorsal incision and approach were used to retrieve the ovaries. Vehicle ( $5 \mu \mathrm{l}$ of PET: $5 \%$ polyethylene glycol, $2.5 \%$ ethanol and $0.8 \%$ Tween- 80 ) was injected into the bursa of one ovary of the pair, and $5 \mu \mathrm{l}$ of a 2-mM stock solution of S1P (Biomol, Plymouth Meeting, PA, USA), prepared in PET, was injected into the bursa of the contralateral ovary. Based on an estimated bursal cavity volume of $50 \mu \mathrm{l}$, the final concentration of S1P in the bursal cavity would have surmounted to approximately $200 \mu \mathrm{M}$. The ovaries were returned to the peritoneal cavity, the incisions were sutured, and the mice were allowed to recover for $3 \mathrm{~h}$ before receiving a single intraperitoneal injection of DXR ( $5 \mathrm{mg} / \mathrm{kg} \mathrm{BW})$. After $24 \mathrm{~h}$, ovaries were collected, coded and processed for histomorphometric evaluation of nonatretic oocyte-containing follicle numbers as previously described. ${ }^{20}$

\section{DXR trafficking in the presence of S1P in vitro}

Mature oocytes were collected as described above. To assess the role of $\mathrm{S} 1 \mathrm{P}$ in the intracellular trafficking of DXR, oocytes were cultured with DXR $(200 \mathrm{nM})$ or with S1P/DXR (S1P, $10 \mu \mathrm{M})$ for up to $2 \mathrm{~h}$, and then fixed with $4 \%$ formalin at various times: 15 or 120 min after exposure. These were stained with DAPI (Sigma) and then viewed on a deconvolution fluorescence microscope. The oocytes were serially scanned, and 10 optical sections were analyzed using DeltaVision software (Applied Precision Inc., CA, USA) as previously described. ${ }^{40}$ As DXR is autofluorescent, the total red fluorescence in the cytoplasm and on chromosomal plate was determined in each section. Average fluorescence from all sections, expressed in RFUs was calculated and presented as a ratio of red/blue (DXR/chromatin content) per oocyte in each group. RFU are arbitrary values for fluorescence intensity, as determined by DeltaVision software. As fluorescent intensities vary from experiment to experiment, the average relative fluorescence for oocytes treated with DXR alone in any given experiment was determined first; this was then considered as $100 \%$ and subsequently values for oocytes treated with S1P + DXR in that experiment were expressed as relative percentages. Results from individual experiments were pooled.

\section{DXR trafficking/retention in oocytes from ASMase- null mice}

To assess the functional role of ceramide in DXR trafficking/retention, we examined oocytes from ASMase-null mice, which are chronically deficient in ceramide generation. ${ }^{24}$ Mature oocytes were collected from ASMasenull and WT females as described above. Oocytes were incubated with DXR for periods up to $1 \mathrm{~h}$; at this time the oocytes were transferred to fresh medium (w/o DXR) and the incubation was allowed to proceed for another $2 \mathrm{~h}$. Oocytes were fixed at various times: 15-120 min after exposure, stained with DAPI (Sigma) and then viewed on a fluorescence microscope. As DXR is autofluorescent, the total red fluorescence in the cytoplasm and on chromosomal plate was determined for each oocyte in the section where the DNA was fully in focus. Average fluorescence, expressed in RFU, in the cytoplasm or in the DNA at each time point was calculated for every genotype. As fluorescent intensities vary from experiment to experiment, three replicates of the experiment were performed the same day within a period of $2 \mathrm{~h}$. The same aliquot of DXR was used for all replicates.

\section{Statistical analysis}

All experiments were independently replicated at least three times with different sets of mice. The combined data from the replicate experiments were subjected to a one-way analysis of variance followed by Scheffe's $F$-test, Fisher's exact test or Student's $t$-test. The results were considered statistically significant at $P<0.05\left(^{*}\right)$ or at $P<0.001\left(^{* *}\right)$. The data in graphs represent the mean \pm S.E.M. of the combined data.

\section{Acknowledgements}

This work was supported by a research grant from the Harvard's Center of Excellence on Women's Health (GIP), by the Department of Physiology at MSU (GIP) and by NIH grant R01-HD34226 (JLT). AJ was supported by $\mathrm{CIHR}$ grant. The CDNA probes used for analysis containing $3^{\prime}$ untranslated region sequence were donated by S Korsmeyer (Bcl-2), S Cory (Bcl-w), K Shibahara (MA-3) and J Reed (Bag-1). Recombinant Bcl- $x_{L \Delta C}$ protein was kindly donated by $D$ Newmeyer.

\section{References}

1. Zenzes MT and Engel W (1978) Ageing of the female gamete. 2 Lactate dehydrogenase activity in fragmented oocytes and ova of the rat. Cytobios 18: 151-156 
2. Fujino $\mathrm{Y}$, Ozaki K, Yamamasu S, Ito F, Matsuoka I, Hayashi E, Nakamura $\mathrm{H}$ Ogita S, Sato $E$ and Inoue M (1996) DNA fragmentation of oocytes in aged mice. Hum. Reprod. 11: 1480-1483

3. Perez Gl, Tao XJ and Tilly JL (1999) Fragmentation and death (a.k.a. apoptosis) of ovulated oocytes. Mol. Hum. Reprod. 5: 414-420

4. Kawahara M, Mori T, Tanaka H and Shimizu H (2002) The suppression of fragmentation by stabilization of actin filament in porcine enucleated oocytes. Theriogenology 58: 1081-1095

5. Miki H, Inoue K, Ogonuki N, Mochida K, Nagashima H, Baba T and Ogura A (2004) Cytoplasmic asters are required for progression past the first cell cycle in cloned mouse embryos. Biol. Reprod. 71: 2022-2028

6. Tilly JL (2001) Commuting the death sentence: how oocytes strive to survive. Nat. Rev. Mol. Cell Biol. 2: 838-848

7. Liu L and Keefe DL (2000) Cytoplasm mediates both development and oxidation-induced apoptotic cell death in mouse zygotes. Biol. Reprod. 62: 1828-1834

8. Liu L, Trimarchi JR and Keefe DL (2000) Involvement of mitochondria in oxidative stress-induced cell death in mouse zygotes. Biol. Reprod. 62: $1745-1753$

9. Trimarchi JR, Liu L, Smith PJ and Keefe DL (2000) Noninvasive measurement of potassium efflux as an early indicator of cell death in mouse embryos. Biol. Reprod. 63: 851-857

10. Perez Gl, Knudson CM, Leykin L, Korsmeyer SJ and Tilly JL (1997) Apoptosisassociated signaling pathways are required for chemotherapy-mediated female germ cell destruction. Nat. Med. 3: 1228-1232

11. Minotti G, Menna P, Salvatorelli E, Cairo G and Gianni L (2004) Anthracyclines: molecular advances and pharmacologic developments in antitumor activity and cardiotoxicity. Pharmacol. Rev. 56: 185-229

12. Gervasoni Jr JE, Fields SZ, Krishna S, Baker MA, Rosado M, Thuraisamy K, Hindenburg AA and Taub RN (1991) Subcellular distribution of daunorubicin in P-glycoprotein-positive and -negative drug-resistant cell lines using laserassisted confocal microscopy. Cancer Res. 51: 4955-4963

13. Coley HM, Amos WB, Twentyman PR and Workman P (1993) Examination by laser scanning confocal fluorescence imaging microscopy of the subcellular localisation of anthracyclines in parent and multidrug resistant cell lines. Br. J. Cancer 67: 1316-1323

14. Tritton TR, Yee $G$ and Wingard Jr LB (1983) Immobilized adriamycin: a tool for separating cell surface from intracellular mechanisms. Fed. Proc. 42: 284-287

15. Serafino A, Sinibaldi-Vallebona P, Pierimarchi P, Bernard P, Gaudiano G, Massa C, Rasi G and Ranagnan G (1999) Induction of apoptosis in neoplastic cells by anthracycline antitumor drugs: nuclear and cytoplasmic triggering? Anticancer Res. 19: 1909-1918

16. Serafino A, Sinibaldi-Vallebona P, Gaudiano G, Koch TH, Rasi G, Garaci E and Ravagnan $G$ (1998) Cytoplasmic localization of anthracycline antitumor drugs conjugated with reduced glutathione: a possible correlation with multidrug resistance mechanisms. Anticancer Res. 18: 1159-1166

17. Clementi ME, Giardina B, Di Stasio E, Mordente A and Misiti F (2003) Doxorubicin-derived metabolites induce release of cytochrome $C$ and inhibition of respiration on cardiac isolated mitochondria. Anticancer Res. 23: 2445-2450

18. Laurent $G$ and Jaffrezou JP (2001) Signaling pathways activated by daunorubicin. Blood 98: 913-924

19. Martin D, Salinas M, Fujita N, Tsuruo T and Cuadrado A (2002) Ceramide and reactive oxygen species generated by $\mathrm{H} 2 \mathrm{O} 2$ induce caspase-3-independent degradation of Akt/protein kinase B. J. Biol. Chem. 277: 42943-42952

20. Morita $Y$, Perez Gl, Paris F, Miranda SR, Ehleiter D, Haimovitz-Friedman A, Fuks Z, Xie Z, Reed JC, Schuchman EH, Kolesnick RN and Tilly JL (2000) Oocyte apoptosis is suppressed by disruption of the acid sphingomyelinase gene or by sphingosine-1-phosphate therapy. Nat. Med. 6: 1109-1114

21. Matikainen T, Perez Gl, Zheng TS, Kluzak TR, Rueda BR, Flavell RA and Tilly JL (2001) Caspase-3 gene knockout defines cell lineage specificity for programmed cell death signaling in the ovary. Endocrinology 142: 2468-2480

22. Bergeron L, Perez Gl, Macdonald G, Shi L, Sun $Y$, Jurisicova A, Varmuza $S$, Latham KE, Flaws JA, Salter JC, Hara H, Moskowitz MA, Li E, Greenberg A,
Tilly $\mathrm{JL}$ and Yuan J (1998) Defects in regulation of apoptosis in caspase-2deficient mice. Genes Dev. 12: 1304-1314

23. Ogretmen B and Hannun YA (2004) Biologically active sphingolipids in cancer pathogenesis and treatment. Nat. Rev. Cancer 4: 604-616

24. Perez Gl, Jurisicova A, Matikainen T, Moriyama T, Kim MR, Takai Y, Pru JK Kolesnick RN and Tilly JL (2005) A central role for ceramide in the age-related acceleration of apoptosis in the female germline. FASEB J. 19: 860-862, E-pub 2005 February 23

25. Liu L, Trimarchi JR, Smith PJ and Keefe DL (2002) Checkpoint for DNA integrity at the first mitosis after oocyte activation. Mol. Reprod. Dev. 62: $277-288$

26. Hinck L, Van Der Smissen P, Heusterpreute M, Donnay I, De Hertogh R and Pampfer S (2001) Identification of caspase-3 and caspase-activated deoxyribonuclease in rat blastocysts and their implication in the induction of chromatin degradation (but not nuclear fragmentation) by high glucose. Biol. Reprod. 64: 555-562

27. Nussbaum DJ and Prather RS (1995) Differential effects of protein synthesis inhibitors on porcine oocyte activation. Mol. Reprod. Dev. 41: 70-75

28. Jurisicova A, Latham KE, Casper RF and Varmuza SL (1998) Expression and regulation of genes associated with cell death during murine preimplantation embryo development. Mol. Reprod. Dev. 51: 243-253

29. Jurisicova A, Antenos M, Varmuza S, Tilly JL and Casper RF (2003) Expression of apoptosis-related genes during human preimplantation embryo development: potential roles for the Harakiri gene product and Caspase- 3 in blastomere fragmentation. Mol. Hum. Reprod. 9: 133-141

30. Bogush T, Smirnova G, Shubina I, Syrkin A and Robert J (1995) Direct evaluation of intracellular accumulation of free and polymer-bound anthracyclines. Cancer Chemother. Pharmacol. 35: 501-505

31. Mimnaugh EG, Fairchild CR, Fruehauf JP and Sinha BK (1991) Biochemical and pharmacological characterization of MCF-7 drug-sensitive and AdrR multidrug-resistant human breast tumor xenografts in athymic nude mice. Biochem. Pharmacol. 42: 391-402

32. Fairchild CR, Ivy SP, Kao-Shan CS, Whang-Peng J, Rosen N, Israel MA, Melera PW, Cowan KH and Goldsmith ME (1987) Isolation of amplified and overexpressed DNA sequences from adriamycin-resistant human breast cancer cells. Cancer Res. 47: 5141-5148

33. Vielhaber G, Pfeiffer S, Brade L, Lindner B, Goldmann T, Vollmer E, Hintze U, Wittern KP and Wepf $R$ (2001) Localization of ceramide and glucosylceramide in human epidermis by immunogold electron microscopy. J. Invest. Dermatol. 117: $1126-1136$

34. Watanabe M, Kitano T, Kondo T, Yabu T, Taguchi Y, Tashima M, Umehara H, Domae N, Uchiyama T and Okazaki T (2004) Increase of nuclear ceramide through caspase-3-dependent regulation of the 'sphingomyelin cycle' in Fas-induced apoptosis. Cancer Res. 64: 1000-1007

35. Veldman RJ, Zerp S, van Blitterswijk WJ and Verheij M (2004) N-hexanoylsphingomyelin potentiates in vitro doxorubicin cytotoxicity by enhancing its cellular influx. Br. J. Cancer 90: 917-925

36. Duty SM, Singh NP, Silva MJ, Barr DB, Brock JW, Ryan L, Herrick RF, Christiani DC and Hauser R (2003) The relationship between environmental exposures to phthalates and DNA damage in human sperm using the neutral comet assay. Environ. Health Perspect. 111: 1164-1169

37. Brady $G$ and Iscove NN (1993) Construction of cDNA libraries from single cells. Methods Enzymol. 225: 611-623

38. Boise LH, Gonzalez-Garcia M, Postema CE, Ding L, Lindsten T, Turka LA, Mao $X$, Nunez $G$ and Thompson CB (1993) bcl-x, a bcl-2-related gene that functions as a dominant regulator of apoptotic cell death. Cell 74: 597-608

39. Kuwana T, Mackey MR, Perkins G, Ellisman MH, Latterich M, Schneiter R, Green DR and Newmeyer DD (2002) Bid, Bax, and lipids cooperate to form supramolecular openings in the outer mitochondrial membrane. Cell 111: 331-342

40. Acton BM, Jurisicova A, Jurisica I and Casper RF (2004) Alterations in mitochondrial membrane potential during preimplantation stages of mouse and human embryo development. Mol. Hum. Reprod. 10: 23-32 